\title{
THE SEVENTEENTH REGULAR MEETING OF THE SOUTHWESTERN SECTION
}

The seventeenth regular meeting of the Southwestern Section of this Society was held at Iowa State College on Saturday, November 29, 1924. The following thirty members of the Society were present at the meeting:

E. S. Allen, Ashton, Birkhoff, Brenke, Chittenden, Coffin, J. T. Colpitts, Daniells, C. W. Emmons, Gaba, Gouwens, Herr, Louis Ingold, J. V. McKelvey, Martha McKelvey, Pattengill, Pierce, J. F. Reilly, H.'L. Rietz, M. M. Roberts, Roever, Rusk, F. R. Smith, Helen Smith, Snedecor, Stouffer, Tappan, J. S. Turner, W. H. Wilson, Roscoe Woods.

Professor E. R. Smith occupied the chair, being relieved during the afternoon session by Professor Birkhoff. The morning session and part of the afternoon session were devoted to the reading of the papers listed below.

During the afternoon session Professor William H. Roever gave a special lecture by invitation of the program committee on Some phases of descriptive geometry. This paper will appear in full in an early issue of this BuLLETIN.

It was voted to hold the next meeting of the Southwestern Section at Kansas City, Missouri, in conjunction with the meeting of the American Association for the Advancement of Science. The following program committee was elected: Professors R. L. Moore (chairman), Louis Ingold, and E. B. Stouffer (secretary).

The titles and abstracts of the papers read are given below. In the absence of the authors the papers by Dr. Green and Professor Moore were read by title.

1. Professor G. D. Birkhoff: On the structure of matter.

The author developes a theory of matter, and electricity attached to matter, on the assumption that the matter is a perfect adiabatic fluid in which every disturbance travels with the velocity of light. 
2. Professor E. W. Chittenden: Note on a compact set which is not perfectly compact.

R. L. Moore has called attention to the fact that the class of transfinite ordinals is compact but not perfectly compact. The present note contains an example of an enumerable set which is compact but not perfectly compact.

3. Professor W. H. Wilson: On two general functional equations.

Several special cases of the functional equations

and

$$
f(x+y)+g(x-y)=h(x) k(y),
$$

$$
F(x+y) G(x-y)=H(x)+K(y),
$$

where $x$ and $y$ are independent, have been considered. The author assumes no relationships between the functions and places no restrictions on them. He expresses these eight functions directly in terms of the functions which satisfy the Cauchy equations $\varphi(x+y)=\varphi(x)+\varphi(y)$ and $\varphi(x+y)=\varphi(x) \varphi(y)$. By virtue of the results obtained in this paper, any information concerning these basic Cauchy equations may be applied immediately to the general equations proposed above or to special cases of these general equations.

4. Professor W. H. Wilson: On a certain functional equation.

The author discusses the functional equation

$f(x+y)-f(x-y)=y g_{k}(x)+y^{3} g_{k-1}(x)+\cdots+y^{2 k-1} g_{1}(x)$, in which $x$ and $y$ are independent (not necessarily real), and $f(x), g_{1}(x), g_{2}(x), \cdots, g_{k}(x)$ are functions to be determined. It is shown that $g_{i}(x)$, for each value $i=1,2, \cdots, k$, satisfies the equation

$$
\sum_{j=0}^{2 i}(-1)^{j}{ }_{2 i} C_{j} g_{i}[x+(2 i-j) y]=0,
$$

where ${ }_{2 i} C_{j}$ is the coefficient of $a^{j}$ in the expansion of $(1+a)^{2 i}$. In addition to this it is obvious that

$$
f(2 x)=f(0)+x g_{k}(x)+x^{3} g_{k-1}(x)+\cdots+x^{2 k-1} g_{1}(x) .
$$

This separation of the unknown functions is effected without restricting the functions (as to continuity, etc.). Equa- 
tions of form (A) have been discussed by the writer (AMERICAN JournaL, vol. 40 (1918), pp. 263 et seq.), in the light of various restrictions on the functions $g_{i}(x)$.

5. Professor J. S. Turner: The spherical analogue of central forces.

If a particle is constrained to move on a sphere, the projection of its acceleration at $P$ upon the tangent plane at $P$ is here called its tangential acceleration. The line of action of the tangential acceleration touches a certain great circle; if $O$ is a point on this great circle, the tangential acceleration at $P$ is said to be directed to $O$. A tangential acceleration which is always directed to a fixed point $O$ is called a central tangential acceleration. It is proved that if a particle moving with a central tangential acceleration directed to $O$ describes a curve on the sphere, then $v \sin p$ is constant, where $v$ is the velocity at $P$, and $p$ is the arcual perpendicular from $O$ on the arcual tangent to the curve at $P$. It is also proved that if the particle describes a spherical conic under a central tangential acceleration directed to a focus $S$, this acceleration is inversely proportional to $\sin ^{2} S P$; and a simple expression is found for the velocity at $P$.

6. Professor J. S. Turner: An extension of the property of the circle known as "Simson's line".

Let $A B C$ be a triangle inscribed in a circle, and let $P, Q$ be two inverse points with respect to the circle. Take $L, M, N$, the images (symmetric points) of $P$ in $B C, C A$, $A B$, and join $Q L, Q M, Q N$, cutting $B C, C A, A B$ in $X, Y, Z$ respectively. Then the points $X, Y, Z$ are collinear.

7. Professor Julia T. Colpitts: Entire functions defined by series of the form $f(z)=\sum f(n)\left(z^{n} / n !\right)$.

In the first part of the paper, $f(n)$ is taken as a polynomial in $n$. It is proved that the necessary and sufficient condition that a function of genus one have a finite number of zeros is that $f(n)$ be the product of $\alpha^{n}(\alpha$ constant) and a polynomial in $n$. In the second part, $f(n)=1 /(n+a)^{s}$, $s>0$ and rational, and $a$ any real number except a negative integer or zero. The nature of the zeros and some approximations to their values are determined. 
8. Professor T. A. Pierce: An explicit solution of a congruence.

A. Hurwitz employed an interpolatory function $(\bmod p)$ in finding the number of real roots of a congruence. Professor Dickson used the same function in constructing a theory of modular invariants. The author shows how the interpolatory function may be used to furnish an explicit solution of a congruence whose modulus is a prime.

9. Dr. C. F. Green: On the summability and regions of summability of a general class of series of the form $\sum c_{n} g(x+n)$.

The first part of the paper is devoted to the determination of the character of the regions of summability of the series for Cesàro type of summability of order $r$. The regions are half planes bounded by straight lines whose abscissas are dependent upon the real part of the exponents in the asymptotic representation of the series. A certain class of auxiliary series (a generalized Dirichlet series) which possesses the same regions of summability as the above series is used as an aid in the determination of the abscissas of summability. Relations between the regions of summability are discussed for the general series and also for particular series of the above type.

10. Professor Louis Ingold: Extensions of the Gauss and Codazzi relations.

Relations connecting certain differential parameters of the first and second orders are obtained which are analogous to the well known relations of Gauss and Codazzi connecting the first and second fundamental quantities of surface theory. The extension to $n$ dimensions is also given.

11. Professor E. S. Allen: The game of "bell and hammer".

The game of "bell and hammer" is begun by the "sale" of five cards at auction. M. Jequier of Neuchatel investigated the probable value of these cards, neglecting the last phase of the game; the present paper completes the investigation. Among other questions, that of the probable number of throws needed to obtain a certain sum exactly (either by one throw or by a succession) is studied. 
12. Professor R. L. Moore: Concerning upper semicontinuous collections of continua which do not separate a given continuum.

A collection of continua $G$ is defined to be an upper semi-continuous collection provided it is true that, if $p$ is a continuum of the collection $G$ and $p_{1}, p_{2}, p_{3}, \ldots$ is a sequence of continua of the collection $G$ such that the lower distance from $p_{n}$ to $p$ approaches zero as a limit as $n$ approaches infinity, then so does the upper distance from $p_{n}$ to $p$. It is shown that if, in a plane $S, M$ is a bounded continuum which does not separate $S$ and $G$ is a countable upper semi-continuous collection of subcontinua of $M$, no one of which separates $M$, then the sum of all the continua of the set $G$ does not separate $M$.

13. Professor R. L. Moore: Concerning sets of segments which cover a point set in the Vitali sense.

In volume 5 of Fundamenta Mathematicae, J. Splawa Neyman showed that if, in space of one dimension, a closed point set $K$ of measure zero is covered in the Vitali sense by a set of segments $G$ then, for every position number $e$, $G$ contains a subset $G_{e}$ such that $G_{e}$ covers $K$ and such that the sum of the lengths of the segments of $G_{e}$ is less than $e$. Sierpinski has raised the question whether this theorem remains true if the requirement that $K$ be closed is omitted. This question here is answered in the negative. But if the requirement in question is replaced by the requirement that there do not exist infinitely many segments of the set $G$, all of length greater than the same positive number, then the resulting proposition is true.

14. Professor F. Reilly: On Lidstone's demonstration of the osculatory interpolation formula.

The author shows that the osculatory interpolation formula of Lidstone can be generalized so that it will involve finite differences of order $2 h+1$, and its graph will have contact of order $k$ with the partial interpolation curves, in a manner analogous to that previously employed in generalizing the formula as demonstrated by Sprague. The two generalized formulas are, in general, not identical.

E. B. STOUFFER, Secretary of the Section. 